\title{
Postneoadjuvant Therapy Stage II Esophageal Adenocarcinoma AJCC v8
}

National Cancer Institute

\section{Source}

National Cancer Institute. Postneoadjuvant Therapy Stage II Esophageal

Adenocarcinoma A/CC v8. NCI Thesaurus. Code C133434.

Stage II includes: T3, N0, M0. T3: Tumor invades adventitia. N0: No regional lymph node metastasis. M0: No distant metastasis. (AJCC 8th ed.) 